\title{
Nonlinear discrete-time systems with delayed control: a reduction
}

\author{
Mattia Mattioni $^{\mathrm{a}, \mathrm{b}}$, Salvatore Monaco ${ }^{\mathrm{a}}$, Dorothée Normand-Cyrot ${ }^{\mathrm{b}}$ \\ ${ }^{a}$ Dipartimento di Ingegneria Informatica, Automatica e Gestionale A. Ruberti (La Sapienza \\ University of Rome); Via Ariosto 25, 00185 Rome, Italy \\ \{mattia.mattioni, salvatore.monaco\}@uniroma1.it. \\ ${ }^{b}$ Laboratoire des Signaux et Systèmes (L2S, CNRS); 3, Rue Joliot Curie, 91192, Gif-sur-Yvette, \\ France mattioni, cyrot\}@l2s. centralesupelec.fr
}

\begin{abstract}
In this work, the notion of reduction is introduced for discrete-time nonlinear input-delayed systems. The retarded dynamics is reduced to a new system which is free of delays and equivalent (in terms of stabilizability) to the original one. Different stabilizing strategies are proposed over the reduced model. Connections with existing predictor-based methods are discussed. The methodology is also worked out over particular classes of time-delay systems as sampled-data dynamics affected by an entire input delay.
\end{abstract}

Keywords: discrete-time systems; nonlinear systems; time-delay systems.

\section{Introduction}

The seminal works by Smith [1] and Artstein [2] have inspired a research toward time-delay systems as an unavoidable paradigm in control theory because their involvement in a lot of practical situations. Investigations have been addressed to the study of the effects of time delay in a control system emphasizing on drawback and also, unexpectedly, advantages. As an example, it has been shown that introducing a delay over the control system might make a non stabilizable (or not controllable) system stabilizable (or controllable) as shown, among others, in [3] or [4]. Furthermore, the huge developments in classical (non-delayed) nonlinear control motivated several important works devoted to extend those well-known results to time-delay systems (e.g., $[3,5,6,7,8]$ and references therein). Nevertheless, a lot of questions still remain unanswered in the case of both continuous and discrete-time dynamics. 
In this paper, the focus is set toward time-delay discrete-time systems which have proven themselves to be of extreme interest for several reasons [9, 10, 11, 12]. Among them, a well-known motivation is provided by the fact that retarded discrete-time systems are finite dimensional so enabling one to restate the design problem over an extended and delay-free state-space model. That is even more interesting when the discrete-time retarded system is issued from the sampling of dynamics affected by input delays [13].

This paper addresses the stabilization of discrete-time nonlinear dynamics affected by input-delay. In this context, several works were carried out, especially in the linear context, by employing descriptor (mostly for linear systems, [3]) or prediction based feedback [14]. As this latter technique usually lacks in robustness, it was recently improved through Immersion and Invariance in [15]. Though, the aforementioned strategy is still hard to extend to larger classes of time-delay systems. Inspired by the work by Artstein [2], we aim at extending the reduction model approach to the discrete-time nonlinear context . Roughly speaking, given a nonlinear discrete-time dynamics affected by a $N$ step input delay, we seek for a model which is delay-free and equivalent to the original retarded system at least as far as stabilizability is concerned. In doing so, we provide an explicit way of computing such a reduction and we prove that any feedback stabilizing its corresponding dynamics also achieves stabilization of the retarded dynamics. Then, we present several ways of designing control by exploiting the properties of the original delay-free system (i.e., the retarded system computed for $N=0$ ) such as smooth stabilizability (in the Lyapunov sense) and $u$-average passivity (in the sense of [16]). Connections to predictor-based feedback laws are established and commented. The cases of Linear Time Invariant (LTI) and input-affine-like dynamics are illustrated as cases study as well as the case of sampled-data systems affected by the so-called entire delay $[17,18]$.

The paper is organized as follows: the problem is formulated in Section 2 and general recalls on discrete-time delay-free systems are provided in Section 3; the definition of the reduction and its stabilizing properties with respect to the original retarded dynamics are in Section 4; the control design is addressed in Section 5 while some case studies are discussed in Section 6; conclusions and perspectives end the paper in Section 7.

Notations and definitions: $\mathbb{N}$ and $\mathbb{R}$ denote, respectively, the set of natural and real numbers including the 0 . For any $u^{j} \in \mathbb{R}$ and $j=1, \ldots, m$ and $w^{i} \in \mathbb{R}$ for a fixed $i \leq m$, we denote $\mathbf{w}^{i}=\left(u^{1}, \ldots, u^{i-1}, w^{i}, 0, \ldots, 0\right) \in \mathbb{R}^{m} \cdot u_{[k-N, k[}$ denotes the story of $u$ over time window $\left[k-N, k\left[\right.\right.$ (i.e., $u_{[k-N, k[}=\left\{u_{k-N}, \ldots, u_{k-1}\right\}$ ). All 
the functions and vector fields defining the dynamics are assumed smooth over the respective definition spaces. $I d$ and $I$ denote the identity function and matrix respectively. Given a vector field $f, L_{f}$ denotes the Lie derivative operator, $\mathrm{L}_{f}=$ $\sum_{i=1}^{n} f_{i}(\cdot) \nabla_{x_{i}}$ with $\nabla_{x_{i}}:=\frac{\partial}{\partial x_{i}}$. Given two vector fields $f$ and $g, a d_{f} g=\mathrm{L}_{f} \circ \mathrm{L}_{g} I d-$ $\mathrm{L}_{g} \circ \mathrm{L}_{f} I d=[f, g]$ and iteratively $a d_{f}^{i} g=\left[f, a d_{f}^{i-1} g\right] . e^{\mathrm{L}_{f}} I d$ (or $e^{f} I d$, when no confusion arises) denotes the associated Lie series operator, $e^{\mathrm{L}_{f}}:=\mathrm{I}+\sum_{i \geq 1} \frac{L_{f}^{i}}{i !}$. Given any smooth function $h: \mathbb{R}^{n} \rightarrow \mathbb{R}$ then $e^{\mathrm{L}_{f}} h(x)=h\left(\left.e^{\mathrm{L}_{f}} \mathrm{Id}\right|_{x}\right)$.

\section{Problem statement}

In this paper, we address the problem of stabilizing via reduction discrete-time dynamics with discrete input delays of the form

$$
x_{k+1}=F\left(x_{k}, u_{k-N}\right)
$$

with $N \in \mathbb{N}, x \in \mathbb{R}^{n}, u \in \mathbb{R}^{m}, F(\cdot): \mathbb{R}^{n} \times \mathbb{R}^{m} \rightarrow \mathbb{R}^{n}$ and the origin as equilibrium to be stabilized. The approach consists in defining a reduction variable (or simply reduction) whose dynamics (the reduced dynamics) is delay-free and of the same dimension as the original retarded system. Moreover, the stabilizability properties of the reduced model are equivalent to those of the original system; namely, any feedback stabilizing the reduced model ensures stabilization of the retarded dynamics as well.

\section{Recalls on discrete-time systems}

In the following, we refer to

$$
\Sigma_{d}: x_{k+1}=F\left(x_{k}, u_{k}\right)
$$

as the delay-free dynamics associated to (1) when $N=0$.

\subsection{The differential-difference (or generically $\left(F_{0}, G\right)$ ) representation}

As proposed in [19], (2) can be equivalently represented by two coupled difference and differential equations whenever the drift term dynamics $F(\cdot, 0):=F_{0}(\cdot)$ 
admits an inverse ${ }^{1}$. More in detail, assuming $m=1, \Sigma_{d}$ described as a map by (2) can be equivalently represented in the $\left(F_{0}, G\right)$-form below

$$
\begin{aligned}
& x^{+}=F_{0}(x), \quad x^{+}:=x^{+}(0) \\
& \frac{\partial x^{+}(u)}{\partial u}=G\left(x^{+}(u), u\right)
\end{aligned}
$$

where $x^{+}(u)$ denotes a curve parametrized by $u$ over $\mathbb{R}^{n}$ and $G(\cdot, u): \mathbb{R}^{n} \times \mathbb{R} \rightarrow$ $\mathbb{R}^{n}$ is satisfied ${ }^{2} G(x, u):=\left.\nabla_{u} F(x, u)\right|_{x=F^{-1}(x, u)}$. It is a matter of computations to verify that for any pair $(x, u)$, the map $F(\cdot, u)$ can be recovered by integrating $(3 \mathrm{~b})$ over $\left[0, u\left[\right.\right.$ with initial condition fixed by (3a) as $x^{+}=F_{0}(x)$. One gets

$$
F(x, u)=x^{+}(u)=F_{0}(x)+\int_{0}^{u} G\left(x^{+}(v), v\right) \mathrm{d} v
$$

and thus $x^{+}\left(u_{k}\right)=x_{k+1}=F\left(x_{k}, u_{k}\right)$ for any pair $\left(x_{k}, u_{k}\right)$.

Remark 3.1. Invertibility of $F_{0}(\cdot)$ guarantees the existence of $G(\cdot, u)$ and integrability of (3b) with well defined solution (4) for u sufficiently close to zero. Invertibility of $F_{0}(\cdot)$ can be relaxed to require the existence of a nominal control value $\bar{u} \in \mathbb{R}$ for which $F(\cdot, \bar{u})$ admits an inverse. In such a case, integrability of $(3 b)$ between $\bar{u}$ and $u$ is still guaranteed for $u$ in a neighborhood of $\bar{u}$.

In the multi-input case $(m>1)$, one defines analogously the $\left(F_{0}, G\right)$-form with $G(x, u)=\left(G^{1}(x, u), \ldots, G^{m}(x, u)\right)$ and $G^{i}(\cdot, u):=\left.\nabla_{u^{i}} F(x, u)\right|_{x=F^{-1}(x, u)}$ for $i=(1, \cdots, m)$ by setting

$$
\begin{aligned}
& x^{+}=F_{0}(x), \quad x^{+}:=x^{+}(0) \\
& \frac{\partial x^{+}(u)}{\partial u^{1}}=G^{1}\left(x^{+}(u), u\right) \\
& \ldots \\
& \frac{\partial x^{+}(u)}{\partial u^{m}}=G^{m}\left(x^{+}(u), u\right) .
\end{aligned}
$$

\footnotetext{
${ }^{1}$ There exists $F_{0}^{-1}: \mathbb{R}^{n} \rightarrow \mathbb{R}^{n}$ such that $F_{0}^{-1}(\cdot) \circ F_{0}(x)=F_{0}(\cdot) \circ F_{0}^{-1}(x)=x$

${ }^{2}$ Given a smooth mapping $F(x, u): \mathbb{R}^{n} \times \mathbb{R}^{m} \rightarrow \mathbb{R}^{n}, F^{-1}(x, u)$ denotes the inverse of $F$ with respect to $x$; i.e., $F\left(F^{-1}(x, u), u\right)=F^{-1}(F(x, u), u)=x$.
} 
The family of controlled vector fields $\left(G^{j}(\cdot, u)\right)_{j=1, \cdots, m}$ verifies by definition the so-called compatibility conditions that guarantee integrability of the so built system of partial derivatives (see [19]). In the multi-input case, (4) generalizes as

$$
F(x, u)=F_{0}(x)+\sum_{i=1}^{m} \int_{0}^{u^{i}} G^{i}\left(x^{+}\left(\mathbf{w}^{i}\right), \mathbf{w}^{i}\right) \mathrm{d} w^{i}
$$

with $\mathbf{w}^{i}=\left(u^{1}, \ldots, u^{i-1}, w^{i}, 0, \ldots, 0\right)$.

As discussed through several contributions (e.g., [20, 21]), the $\left(G^{j}(\cdot, u)\right)_{j=1, \cdots, m}$ provide a differential geometric apparatus to analyze and formulate in an elegant way the properties of nonlinear discrete-time dynamics and their associated flows. Some of the aspects that are instrumental in the present context are recalled below when $m=1$ with intuitive extension to $m>1$.

At first, given $G(\cdot, u)$, one defines $A d_{F_{0}} G(\cdot, u)$ as its transport along the drift term $F_{0}(\cdot)$ as $($ see $[19,21])$

$$
A d_{F_{0}} G(x, u):=\left[\nabla_{x} F_{0}(x)\right]_{F_{0}^{-1}(x)} G\left(F_{0}^{-1}(x), u\right) .
$$

Iteratively, one sets $A d_{F_{0}}^{i} G(x, u):=A d_{F_{0}} \circ A d_{F_{0}}^{i-1} G(x, u)$ with $A d_{F_{0}}^{0} G(x, u):=G(x, u)$. Given any smooth mapping $S(\cdot): \mathbb{R}^{n} \rightarrow \mathbb{R}$, a useful outcome of the $\left(F_{0}, G\right)$ representation is to split the evolution of $S(\cdot)$ along the dynamics (2) into the free (or uncontrolled) and forced contributions; namely, one writes

$$
S(F(x, u))=S\left(F_{0}(x)\right)+\int_{0}^{u} \mathrm{~L}_{G(\cdot, v)} S\left(x^{+}(v)\right) \mathrm{d} v .
$$

This is useful in the definition of $u$-average passivity that is recalled below [16].

\section{2. u-average passivity and stabilization}

The notion of u-average passivity has been introduced in discrete time in [16]. First, consider the case of a single-input system (i.e., when $m=1$ ).

Definition 3.1. $\Sigma_{d}$ with $u \in \mathbb{R}$ and output $H(\cdot)$ is $u$-average passive (or average passive) if there exists a positive semi definite function $S(\cdot): \mathbb{R}^{n} \rightarrow \mathbb{R}_{\geq 0}$, the storage function, such that for any pair $\left(x_{k}, u_{k}\right), k \geq 0$, one verifies the inequality

$$
S\left(F\left(x_{k}, u_{k}\right)\right)-S\left(x_{k}\right) \leq H_{a v}\left(x_{k}, u_{k}\right) u_{k}
$$


where $H_{a v}(x, u)$ denotes the $u$-average output mapping associated with $H(x)$; i.e.

$$
H_{a v}(x, u):=\frac{1}{u} \int_{0}^{u} H\left(x^{+}(v)\right) \mathrm{d} v
$$

with $H_{a v}(x, 0)=H\left(x^{+}(0)\right)=H\left(F_{0}(x)\right)$.

According to (8), the dissipativative inequality (9) rewrites as

$$
S\left(F_{0}\left(x_{k}\right)\right)-S\left(x_{k}\right)+\int_{0}^{u_{k}} \mathrm{~L}_{G(\cdot, v)} S\left(x^{+}(v)\right) \mathrm{d} v \leq \int_{0}^{u_{k}} H\left(x^{+}(v)\right) \mathrm{d} v
$$

with by definition $\int_{0}^{u_{k}} H\left(x^{+}(v)\right) \mathrm{d} v:=H_{a v}\left(x_{k}, u_{k}\right) u_{k}$.

Remark 3.2. u-average passivity can be generalized to systems with direct inputoutput link $H(\cdot, u)$ smoothly parameterized by $u$ thus setting

$$
H_{a v}(x, u):=\frac{1}{u} \int_{0}^{u} H\left(x^{+}(v), v\right) \mathrm{d} v
$$

with $H_{a v}(x, 0)=H\left(x^{+}(0), 0\right)=H\left(F_{0}(x), 0\right)$.

We recall the notion of zero-state detectability.

Definition 3.2. Let $\Sigma_{d}$ with output $H(\cdot, u)$ and let $\mathscr{Z} \subset \mathbb{R}^{n}$ be the largest positively invariant set contained in $\left\{x \in \mathbb{R}^{n} \mid H(x, 0)=0\right\}$. We say that $\Sigma_{d}$ is Zero-StateDetectable $(Z S D)$ if $x=0$ is asymptotically stable conditionally to $\mathscr{Z}$.

The following result extends $u$-average passivity to the case $m>1$.

Theorem 3.1. Consider $\Sigma_{d}$ with $m \geq 1$ and assume the existence of a positive definite storage function $S(\cdot): \mathbb{R}^{n} \rightarrow \mathbb{R}$ such that $S\left(F_{0}(x)\right)-S(x) \leq 0$. Then,

(i). $\Sigma_{d}$ with output $H(x, u)=\left(\mathrm{L}_{G(\cdot, u)} S(x)\right)^{\top}$ is u-average passive; i.e., the dissipativity inequality holds

$$
S\left(x^{+}(u)\right)-S(x) \leq H_{a v}^{\top}(x, u) u=\sum_{i=1}^{m} H_{a v}^{i}(x, u) u^{i}
$$

with

$$
H_{a v}^{i}(x, u)=\frac{1}{u^{i}} \int_{0}^{u^{i}} \mathrm{~L}_{G^{i}\left(\cdot, \mathbf{w}^{i}\right)} S\left(x^{+}\left(\mathbf{w}^{i}\right)\right) \mathrm{d} w^{i}
$$


(ii). If $\Sigma_{d}$ with output $H(x, u)$ is ZSD, then any feedback $u=\gamma(x)$ solving

$$
u+K H_{a v}(x, u)=0, \quad \text { with } K>0
$$

achieves global asymptotic stabilization of the equilibrium of $\Sigma_{d}$.

Accordingly, the feedback satisfying (12) is an $u$-average passivity based controller ( $\mathrm{uAvPBC)}$ that we shall refer to as the negative u-average output feedback for discrete-time systems in the form of (3) with output $H(\cdot, u)$.

Remark 3.3. The feedback $u=\gamma(x)$ is defined as the implicit solution to the nonlinear equality (12) which is hard to solve in practice. Nevertheless, an approximate and bounded solution still yielding GAS of the closed-loop equilibrium was proposed in [22, 23] and takes the form $\gamma(x)=-\hat{K}(x) H_{a v}(x, 0)$ with a suitable dynamical gain $\hat{K}(x)>0$ and $H_{a v}(x, 0)=H\left(F_{0}(x), 0\right)$.

\section{Reduction of time-delay systems}

Considering now the input-delayed dynamics (1) with invertible drift $F_{0}(x)$, we show how to recast the problem of stabilizing (1) into the one of stabilizing a delay-free dynamics of the form $\eta_{k+1}=F_{r}\left(N, \eta_{k}, u_{k}\right): \mathbb{N} \times \mathbb{R}^{n} \times \mathbb{R}^{m} \rightarrow \mathbb{R}^{n}$ for a suitably defined reduction variable $\eta_{k}:=r\left(x_{k}, u_{[k-N, k]}\right): \mathbb{R}^{n} \times\left\{\mathbb{R}^{m}\right\}^{N} \rightarrow \mathbb{R}^{n}$.

Theorem 4.1. Consider the dynamics (1) with invertible drift term $F_{0}(\cdot)$. Then,

$$
\eta_{k}=F_{0}^{-N}(\cdot) \circ F^{N}\left(x_{k}, u_{[k-N, k]}\right)
$$

with

$F^{N}\left(x_{k}, u_{[k-N, k]}\right)=F^{N-1}\left(\cdot, u_{[k-N+1, k]}\right) \circ F\left(x_{k}, u_{k-N}\right)=F\left(\cdot, u_{k-1}\right) \circ \cdots \circ F\left(x_{k}, u_{k-N}\right)$ $F^{1}\left(x_{k}, u_{[k-1, k]}\right)=F\left(x_{k}, u_{k-1}\right)$

is a reduction for (1) evolving according to the reduced model

$$
\eta_{k+1}=F_{r}\left(N, \eta_{k}, u_{k}\right)
$$

with $F_{r}(N, \eta, u):=F_{0}^{-N}(\cdot) \circ F(\cdot, u) \circ F_{0}^{N}(\eta)$ or, equivalently

$$
\begin{aligned}
& \eta^{+}=F_{0}(\eta), \quad \eta^{+}:=\eta^{+}(0) \\
& \frac{\partial \eta^{+}(u)}{\partial u}=A d_{F_{0}^{-N}} G\left(\eta^{+}(u), u\right) .
\end{aligned}
$$


Proof: In order to show the result, one computes

$$
\begin{aligned}
\eta_{k+1} & =F_{0}^{-N}(\cdot) \circ F^{N}\left(\cdot, u_{[k-N+1, k+1[}\right) \circ F\left(x_{k}, u_{k-N}\right) \\
& =F_{0}^{-N}(\cdot) \circ F\left(\cdot, u_{k}\right) \circ F^{N}\left(x_{k}, u_{[k-N, k]}\right) .
\end{aligned}
$$

By rewriting $x_{k}=F^{-N}\left(\cdot, u_{[k-N, k]}\right) \circ F_{0}^{N}\left(\eta_{k}\right)$ and substituting it into (16) one gets the result. $\triangleleft$

The representation (15) of the reduced model emphasizes its geometric structure: the free evolution (15a) is unchanged while the forced component (15b) (which is actually affected by the delay) is transported backward along the drift dynamics composed $N$ times. As a result, the reduced system is delay free over the input but explicitly parametrized by the delay $N$.

Now, the problem of stabilizing the retarded system (1) is recast into the one of stabilizing the equilibrium reduced dynamics (14).

Theorem 4.2. Consider the dynamics $(1)$ with $F_{0}(\cdot)$ invertible and reduced model (14). Then, any feedback $u=\alpha\left(\eta_{k}\right)$ such that $\alpha(0)=0$ ensuring GAS of the equilibrium of (14) achieves GAS of the equilibrium of (1) in closed-loop. Furthermore, if $\eta_{k}=0$ for $k \geq \bar{k}$, then $x_{k}=0$ for $k \geq \vec{k}+N+1$ and, thus, converges to zero in finite time.

Proof: Introduce the auxiliary state $v_{i k}=u_{k-N+1-i}$ so that $v_{i k+1}=v_{i+1 k}$ for $i=1, \ldots, N$. Because $F_{0}(x)$ is invertible, $F(x, u)$ is locally invertible so that one can introduce the cascade system

$$
\begin{aligned}
& x_{k+1}=F_{1}\left(\eta_{k}, v_{k}\right) \\
& v_{k+1}=A_{0} v_{k}+B_{0} u_{k} \\
& \eta_{k+1}=F_{r}\left(N, \eta_{k}, u_{k}\right)
\end{aligned}
$$

with $v_{k}=\left(v_{1 k}, \ldots, v_{N k}\right)^{\top}, v_{j k}=\operatorname{col}\left(v_{j k}^{1}, \ldots, v_{j k}^{m}\right)$ for $i, j=1, \ldots, N$ and

$$
\begin{aligned}
& F_{1}\left(\eta_{k}, v_{k}\right)=\left.F^{-(N-1)}\left(\cdot, u_{[k-N+1, k-1[}\right) \circ F_{0}^{N}\left(\eta_{k}\right)\right|_{u_{k-N+1-i}=v_{i k}} \\
& A_{0}=\left(\begin{array}{cc}
\mathbf{0}_{m(N-1) \times m} & D \\
\mathbf{0}_{m \times m} & \mathbf{0}_{m \times m(N-1)}
\end{array}\right) \\
& D=\operatorname{diag}\left\{I_{m \times m}, \ldots, I_{m \times m}\right\}, \quad B_{0}=\left(\begin{array}{ll}
\mathbf{0}^{\top} & I_{m \times m}
\end{array}\right)^{\top} .
\end{aligned}
$$

By exploiting the strict feedforward structure [24], one gets the result. $\triangleleft$ 
We note that methodologies involving a suitable dynamical state extension over the delayed inputs transform the system into an equivalent one where the effect of the delay is explicitly hidden $[3,13,14]$. As a matter of fact, the corresponding augmented dynamics is free of delays in the control but also in the mapping characterizing the evolutions. The corresponding design is then lead to stabilizing the extended dynamics which is apparently free of any delay. In the case of reduction, the controlled component of the reduced dynamics is explicitly parametrized by $N$. This explicit dependence might be exploited to directly infer control laws which take advantage of the properties of the uncontrolled systems (e.g., passivity) while possibly guaranteeing robustness with respect to variations of $N$ within a fixed range of values. Moreover, the reduced dynamics preserves the same dimension as the original retarded system.

Remark 4.1. Since here the problem of stabilizing the origin of (1) is addressed, the reduction is directly computed over the x-dynamics. However, this method extends to a larger variety of control problems that can be turned into the one of stabilizing the origin of a suitably defined dynamics (e.g., tracking, regulation). In those cases, one deduces the reduction over the dynamics defining the control objective. As an example, in the case of tracking of a reference signal $r$, one should compute the reduction over the error dynamics $\varepsilon_{k}=x_{k}-r_{k}$ and then stabilize the origin of the reduced error model.

\section{Control design}

In the following we present two stabilizing feedback strategies over the reduced model designed upon the notion of Discrete Input-Lyapunov Matching (DILM) and $u$-average Passivity ( $u$-AvPB) respectively. A comparison with a purely Prediction-Based (PB) strategy is discussed.

\subsection{Stabilization via D-ILM}

The following standing assumption is set.

Assumption 5.1. The delay-free dynamics (2) (equivalently (3)) is smoothly stabilizable; i.e., there exists a smooth feedback $u_{k}=\gamma\left(x_{k}\right): \mathbb{R}^{n} \rightarrow \mathbb{R}^{m}$ and radially unbounded and positive definite Lyapunov function $V: \mathbb{R}^{n} \rightarrow \mathbb{R}_{\geq 0}$ such that $\Delta_{k} V\left(x_{k}\right):=V\left(F\left(x_{k}, \gamma\left(x_{k}\right)\right)\right)-V\left(x_{k}\right)<0$ and $\operatorname{rank}\left\{\mathrm{L}_{G(\cdot, 0)} V\left(F_{0}\left(x_{k}\right)\right)\right\}=1$ whenever $x_{k} \neq 0$. 
In the delay free case $N=0, \eta \equiv x$ and thus $F_{r}(0, \eta, u)=F(x, u)$ so that the feedback $\gamma(\cdot)$ defined in Assumption 5.1 is clearly stabilizing for the reduced dynamics so that $\eta_{k+1}=F_{r}\left(0, \eta_{k}, \gamma\left(\eta_{k}\right)\right)$ has a GAS equilibrium at the origin with strictly decreasing Lyapunov function $V(\eta)$. Thus, for a generic $N>0$, the idea is to look for the stabilizing control $u_{k}=L_{r}\left(N, \eta_{k}\right)$ which satisfies the Input Lyapunov Matching equality at any time instant; i.e. $\forall \eta_{k}, k \geq 0, u_{k}$ is such that

$$
V\left(F_{r}\left(N, \eta_{k}, u_{k}\right)\right)-V\left(\eta_{k}\right)=V\left(F_{r}\left(0, \eta_{k}, \gamma\left(\eta_{k}\right)\right)-V\left(\eta_{k}\right)\right.
$$

which simplifies as

$$
V\left(F_{r}\left(N, \eta_{k}, u_{k}\right)\right)-V\left(F_{r}\left(0, \eta_{k}, \gamma\left(\eta_{k}\right)\right)=0, \quad \forall k \geq 0\right.
$$

Theorem 5.1. Consider the retarded dynamics (1) and the corresponding reduced model (14). If the delay-free dynamics (2) verifies Assumption 5.1, then the feedback $u_{k}=L_{r}\left(N, \eta_{k}\right)$ computed as the solution to the D-ILM equality (17) ensures GAS of the equilibrium of (14). As a consequence, the aforementioned feedback globally asymptotically stabilizes the closed-loop equilibrium of (1).

Proof: $\quad$ From Assumption 5.1, one has $V\left(F_{r}\left(0, \eta_{k}, \gamma\left(\eta_{k}\right)\right)-V\left(\eta_{k}\right)<0, \quad \forall k \geq\right.$ 0 and thus, because of matching $V\left(F_{r}\left(N, \eta_{k}, L_{r}\left(N, \eta_{k}\right)\right)\right)-V\left(\eta_{k}\right)<0, \quad \forall k \geq 0$ Thus, GAS of the equilibrium of (14) follows by construction while stability of (1) comes from direct application of Theorem 4.2. Existence of a solution to the above equality is ensured by the fact that $\operatorname{rank}\left\{\mathrm{L}_{G(\cdot, 0)} V\left(F_{0}(x)\right)\right\}=1$ for any $x \neq 0$ as it implies $\operatorname{rank}\left\{\mathrm{L}_{\bar{G}_{N}(\cdot, 0)} V\left(F_{0}(\eta)\right)\right\}=1$ for $\eta \neq 0$. $\triangleleft$

Remark 5.1. The rank condition in Assumption 5.1 is necessary for proving (through suitably invoking the implicit function theorem) the existence of a solution to the implicit equality (17) that rewrites as a former series expansion in powers of $N$. Such a series is invertible if the rank condition holds for $N=0$ and $u=0$. In that case, the solution takes the form of an asymptotic series expansion in powers of $N$ around the delay-free solution $u_{k}=\gamma\left(\eta_{k}\right)$ (i.e., $u=\gamma(\eta)+\sum_{i>0} \frac{N^{j}}{(j+1) !} \gamma^{j}(\eta)$ ).

When $u \in \mathbb{R}$, it is a matter of computation to rewrite (17) as

$$
\int_{0}^{u} \mathrm{~L}_{\bar{G}_{N}(\cdot, v)} V\left(F_{r}(N, \eta, v)\right) \mathrm{d} v=\int_{0}^{\gamma(\eta)} \mathrm{L}_{G(\cdot, v)} V(F(\eta, v)) \mathrm{d} v
$$


so specifying the control $u=L_{r}(N, \eta)$ as

$$
L_{r}(N, \eta)=K(N, \eta, u) \gamma(\eta)
$$

with $K(N, \eta, u)$ solution of the implicit equality

$$
K(N, \eta, u)=\left(\int_{0}^{1} \mathrm{~L}_{\bar{G}_{N}(\cdot, s u)} V\left(F_{r}(N, \eta, s u)\right) \mathrm{d} s\right)^{-1} \int_{0}^{1} \mathrm{~L}_{G(\cdot, s \gamma(\eta))} V(F(\eta, s \gamma(\eta))) \mathrm{d} s .
$$

It is important to note that one recovers the delay-free feedback when setting in the above equation $N=0$ so getting $K(0, \eta, \gamma(\eta)))=1$. This can be easily extended to the multi-input case along the same lines

Remark 5.2. The D-ILM equality (17) is approximately solved ${ }^{3}$ in $O\left(|u|^{2}\right)$ by setting $u_{\text {app }}=K(N, \eta, 0) \gamma(\eta)$.

\subsection{Stabilization via average passivity}

In this part, the following assumption is set.

Assumption 5.2. Considering the delay-free dynamics (2) (equivalently (3)), there exists a proper and positive definitive $S(\cdot): \mathbb{R}^{n} \rightarrow \mathbb{R}$ such that $S\left(F_{0}(x)\right)-S(x) \leq 0$.

As a consequence, the following result can be proven.

Theorem 5.2. Consider the retarded dynamics (1) with invertible $F_{0}(\cdot)$ and the corresponding reduced model (14). If the delay-free dynamics (2) verifies Assumption (5.2), then the following holds true:

(i) The reduced model (14) is u-average passive with output

$$
H(N, \eta, u)=\left(\mathrm{L}_{\bar{G}_{N}(\cdot, u)} S(\eta)\right)^{\top}
$$

(ii) If the reduced model (14) is ZSD with respect to $H(N, \cdot, u)=\left(\mathrm{L}_{\bar{G}_{N}(\cdot, u)} S(\cdot)\right)^{\top}$ then, the feedback solution to

$$
u+K H_{a v}(N, \eta, u)=0, \quad K>0
$$

\footnotetext{
${ }^{3}$ A function $R(\eta, u)=O\left(|u|^{p}\right)$ is said of order $|u|^{p} ; p \geq 1$ if whenever it is defined it can be written as $R(x,|u|)=|u|^{p-1} \tilde{R}(x,|u|)$ and there exist a function $\theta \in \mathscr{K}_{\infty}$ and $|u|^{*}>0$ s. t. $\forall|u| \leq|u|^{*}$, $|\tilde{R}(x,|u|)| \leq \theta(|u|)$.
} 
with $H_{a v}(N, \eta, u)=\left(H_{a v}^{1}(N, \eta, u) \ldots H_{a v}^{m}(N, \eta, u)\right)^{T}$ and, for $i=1, \ldots, m$

$$
H_{a v}^{i}(N, \eta, u)=\frac{1}{u^{i}} \int_{0}^{u^{i}} \mathrm{~L}_{\bar{G}_{N}^{i}\left(\cdot, \mathbf{w}^{i}\right)} S\left(\eta^{+}\left(\mathbf{w}^{i}\right)\right) \mathrm{d} w^{i}
$$

ensures GAS of the closed-loop equilibrium of (14) and, hence, of (1).

Proof: Item (i) directly follows from $S\left(F_{0}(\eta)\right)-S(\eta)<0$ since by definition

$$
\begin{aligned}
S\left(\eta_{k+1}\right)-S\left(\eta_{k}\right) & =S\left(F_{0}\left(\eta_{k}\right)\right)-S\left(\eta_{k}\right)+\sum_{i=1}^{m} \int_{0}^{u^{i}} \mathrm{~L}_{\bar{G}_{N}^{i}\left(\cdot, \mathbf{w}^{i}\right)} S\left(\eta^{+}\left(\mathbf{w}^{i}\right)\right) \mathrm{d} w^{i} \\
& \leq H_{a v}^{\top}\left(N, \eta_{k}, u_{k}\right) u_{k} .
\end{aligned}
$$

Concerning (ii), from ZSD of the reduced dynamics with output $\left(\mathrm{L}_{\bar{G}_{N}(\cdot, 0)} S(\cdot)\right)^{\top}$ the result follows from Theorem 3.1. $\triangleleft$

When $u \in \mathbb{R}$, the solution $u=L_{a v}(N, \eta)$ solution to (20) rewrites as

$$
L_{a v}(N, \eta)=-\int_{0}^{1} \mathrm{~L}_{\bar{G}_{N}\left(\cdot, s L_{a v}(N, \eta)\right)} V\left(F_{r}\left(N, \eta, s L_{a v}(N, \eta)\right)\right) \mathrm{d} s
$$

which recovers when $N=0$ the delay-free solution

$$
L_{a v}(N, \eta)=-\int_{0}^{1} \mathrm{~L}_{G\left(\cdot, s L_{a v}(N, \eta)\right)} V\left(F\left(\eta, s L_{a v}(N, \eta)\right)\right) \mathrm{d} s .
$$

Remark 5.3. Approximate and bounded solutions to (17)-(20) can be explicitly computed by exploiting the result in [22, 23] as pointed out in Remark 3.3.

An academic example: Consider the retarded dynamics

$$
x_{1 k+1}=e^{\frac{2}{3} x_{2 k}+\frac{1}{2} u_{k-N}} x_{1 k}, \quad x_{2 k+1}=\frac{1}{3} x_{2 k}+u_{k-N} .
$$

It is a matter of computations to verify that when $N=0$, the delay-free system is described by the $\left(F_{0}, G\right)$ representation

$$
x_{1}^{+}=e^{\frac{2}{3} x_{2}} x_{1}, \quad \frac{\partial x_{1}^{+}(u)}{\partial u}=\frac{1}{2} x_{1}^{+}(u), \quad x_{2}^{+}=\frac{1}{3} x_{2}, \quad \frac{\partial x_{2}^{+}(u)}{\partial u}=1
$$

and verifies Assumption 5.2 with storage function $S(x)=\frac{1}{2}\left(e^{2 x_{2}} x_{1}^{2}+x_{2}^{2}\right)$ so that $S\left(F_{0}(x)\right)-S(x)=-\frac{4}{9} x_{2}^{2} \leq 0$. 
Assume now $N=1$ and define the reduction variable $\eta=\left(\eta_{1}, \eta_{2}\right)^{\top}$ as

$$
\eta_{1 k}=e^{-\frac{3}{2} u_{k-1}} x_{1 k}, \quad \eta_{2 k}=x_{2 k}+3 u_{k-1}
$$

evolving according to the dynamics

$$
\eta_{1}^{+}=e^{\frac{2}{3} \eta_{2}} \eta_{1}, \quad \frac{\partial \eta_{1}^{+}(u)}{\partial u}=-\frac{3}{2} \eta_{1}^{+}(u), \quad \eta_{2}^{+}=\frac{1}{3} \eta_{2}, \quad \frac{\partial \eta_{2}^{+}(u)}{\partial u}=3 .
$$

As a direct consequence of Theorem 5.2, (24) is $u$-average passive with respect to output $H(1, \eta)=\frac{3}{2} e^{2 \eta_{2}} \eta_{1}^{2}+3 \eta_{2}$ and average

$$
H_{a v}(1, \eta, u)=\eta_{2}+\frac{9}{2} u+\frac{1}{2} e^{2 \eta_{2}} \eta_{1}^{2} \frac{e^{3 u}-1}{u}
$$

that are computed through the same storage function $S(\cdot)$ as in the delay-free case. Thus, the feedback $u=L_{a v}(N, \eta)$ solution to the implicit equality

$$
u=\varphi(\eta, u)=-\frac{2}{11} \eta_{2}-\frac{1}{11} e^{2 \eta_{2}} \eta_{1}^{2} \frac{e^{3 u}-1}{u}
$$

ensures GAS of the closed-loop equilibrium. The equality (25) is highly nonlinear in the control $u$ and is approximatively solved in $O\left(|u|^{2}\right)$ by

$$
u_{\text {app }}(\eta)=\lim _{u \rightarrow 0} \varphi(\eta, u)=-\frac{2}{11} \eta_{2}-\frac{3}{11} e^{2 \eta_{2}} \eta_{1}^{2}
$$

Though, the above solution only ensures stability of the equilibrium as long as $u$ is bounded and closed to 0 . To overcome this issue, according to Remark 3.3, we compute an approximate solution to (25) which is bounded and globally asymptotically stabilizing. It takes the form

$$
\bar{u}(\eta)=\hat{K}(\eta) u_{a p p}(\eta)
$$

with $|\bar{u}(\eta)| \leq \mu$ for any fixed positive $\mu \in \mathbb{R}$ and $\hat{K}(\eta) \in] 0, C(\eta)[$ where tha mappings

$C\left(\eta_{k}\right)=\frac{\mu}{(1+2 \mu)\left(1+\left|u_{a p p}\left(\eta_{k}\right)\right|\right)} S(\eta), \quad S\left(\eta_{k}\right)=\min _{|u| \leq \frac{1}{2}}\left\{1, \frac{|u|}{\left|\varphi\left(\eta_{k}, u\right)-u_{a p p}\left(\eta_{k}\right)\right|}\right\}$

are computed at any time instant $k \geq 0$. 


\subsubsection{Reduction vs Prediction}

Assumption 5.1 guarantees also the existence of a prediction-based feedback that stabilizes the origin of (1) in closed-loop. As a matter of fact, defining as usual the prediction state $z_{k}=F^{N}\left(x_{k}, u_{[k-N, k]}\right)$, one gets the predictor dynamics

$$
z_{k+1}=F\left(z_{k}, u_{k}\right)
$$

which coincides with the delay free one. As a consequence, by applying the feedback $\gamma(\cdot)$ in Assumption 5.1 over $z$ (i.e., setting $u_{k}=\gamma\left(z_{k}\right)$ ) ensures stabilization of the predictor dynamics and, thus, of the retarded system (1). It turns out, that the above prediction-based feedback can be interpreted a particular case of reductionbased system because $z_{k}=F_{0}^{N}\left(\eta_{k}\right)$ so that $\gamma\left(z_{k}\right)$ rewrites in terms of reduction as $u_{k}=\gamma\left(F_{0}^{N}\left(\eta_{k}\right)\right)$. Accordingly, the existence of a stabilizing prediction-based feedback for input delayed dynamics (1) implies the existence a reduction-based one.

Remark 5.4. We note that by construction the prediction-based feedback $\gamma(z):=$ $\gamma\left(F^{N}\left(x_{k}, u_{[k-N, k[}\right)\right)$ ensures Input-Lyapunov Matching of the closed loop delay free dynamics with $N$ step delays (i.e., at step $k+N$ ) while the reduction-based feedback proposed in Section 5.1 guarantees Input-Lyapunov Matching of the closed loop delay free dynamics without any delay (i.e., at step $k$ ).

Remark 5.5. Because of the mere compensation purpose, the prediction-based feedback $u=\gamma\left(z_{k}\right)$ lacks in robustness with respect to prediction error and uncertainty over the delay length. This issue was discussed in [15] in the context of Immersion and Invariance (I\&I) by also exploiting a suitable dynamical extension that makes (1) delay-free. Roughly speaking, the I\&I feedback $u_{k}=\gamma\left(z_{k}\right)-\mathrm{L}\left(z_{k}\right) e_{k}$ adds a proportional term over the prediction error for a suitable dynamic gain $\mathrm{L}(\cdot)$ and prediction error $e_{k}=\operatorname{col}\left\{e^{1}, \ldots, e^{N}\right\}$ with $e_{k}^{i}=$ $v_{k}^{i}-\gamma\left(x_{k+i-1}\right)$ and $v_{k}^{i}=u_{k-N+i-1}$ for $i=1, \ldots, N$.

\section{Case studies}

\subsection{LTI systems}

Consider the case of linear time-invariant (LTI) systems of the form

$$
x_{k+1}=A x_{k}+B u_{k-N}
$$


then, (13) specifies as

$$
\eta_{k}=x_{k}+\sum_{j=k-N}^{k-1} A^{k-1-N-j} B u_{j}
$$

and evolves according to

$$
\eta_{k+1}=A \eta_{k}+A^{-N} B u_{k}
$$

so that controllability of $\left(A, A^{-N} B\right)$ is enough to ensure the existence of a reductionbased feedback. For, the following result is proven.

Proposition 6.1. Consider the LTI system (27) and let (28) be a reduction with model (29). Then, (29) is controllable if and only if

(i) the couple $(A, B)$ is controllable;

(ii) A has no zero eigenvalue.

As a consequence, any feedback $u=L x$ ensuring that $A+A^{-N} B L$ is Schur asymptotically stabilizes (27).

Proof: In order to show the result, one has to prove that the above conditions are necessary and sufficient to guarantee that the matrix

$$
\mathscr{R}_{N}=\left(A^{-N} B: A^{-N+1} B \vdots \quad \ldots \quad \vdots A^{n-1-N} B\right)
$$

is full-rank $n$. For, we rewrite $\mathscr{R}_{N}=A^{-N} \mathscr{R}$ where $\mathscr{R}$ denotes the controllability matrix of the delay-free system, $\mathscr{R}=\left(B \vdots A B \vdots \ldots \quad \ldots A^{n-1} B\right)$. Proving that $\mathscr{R}_{N}$ corresponds to prove that $\operatorname{Ker}\left\{A^{-N}\right\} \cap \operatorname{Im}\{\mathscr{R}\} \equiv\{0\}$. The sufficiency of (i) and (ii) is straightforward as (ii) implies that $A^{-N}$ is non singular and, thus, $\operatorname{Ker}\left\{A^{-N}\right\} \equiv\{0\}$. The necessity can be easily proven by contradiction by assuming that $\operatorname{Ker}\left\{A^{-N}\right\} \cap \operatorname{Im}\{\mathscr{R}\}-\{0\} \neq 0$ so that there exists $\bar{x} \in \operatorname{Im}\{\mathscr{R}\}-\{0\}$ such that $A^{-N} \bar{x}=0$. Because of (i), one has that $\bar{x}=0$, so contradicting the assumption. Thus, one has that any feedback that $u=L x$ ensuring that $A+A^{-N} B L$ is Schur asymptotically stabilizes (29). Finally, to prove asymptotic stability of (27), one introduces the auxiliary state $v_{i k}=u_{k-N+1-i}$ (for $i=1, \ldots, N$ ) so that $v_{i k+1}=v_{i+1 k}$ and consider the upper-triangular system

$$
x_{k+1}=\hat{A} v_{k}+A \eta_{k}, \quad v_{k+1}=A_{0} v_{k}+B_{0} L \eta_{k}, \quad \eta_{k+1}=\left(A+A^{-N} B L\right) \eta_{k}
$$


that is clearly asymptotically stable, so concluding the proof. $\triangleleft$

Proposition 6.1 shows that the problem of stabilizing the retarded system (27) is reformulated as an eigenvalue placement problem over the reduced model (29) provided that $A$ is invertible.

One can assign the spectrum of $A+A^{-N} B L$ as directly dependent on $N$ so to guarantee stability for a fixed range of $N$. In this sense, one does not fully compensate the effect of the delay over the closed-loop system but might ensure that the closed-loop eigenvalues are stable for a fixed range of values of $N$ so possibly improving robustness.

Remark 6.1. Asking for A to be invertible seems to be only a sufficient requirement as non invertibility of A corresponds to the presence of 0 eigenvalues corresponding to asymptotically stable modes. Thus, one might still define a suitable reduction over a lower dimensional state-space by leaving the stable part (associated to the 0 eigenvalues) unchanged.

Remark 6.2. Assumption ( $i$ ) in Proposition 6.1 can be weakened to requiring only stabilizability of the couple $(A, B)$ without affecting the result.

\subsection{Input-affine-like dynamics}

Consider the class of time-delay system (1) whose delay-free $\left(F_{0}, G\right)$ representation is provided by [16]

$$
\begin{aligned}
& x^{+}=F_{0}(x), \quad x^{+}:=x^{+}(0) \\
& \frac{\partial x^{+}(u)}{\partial u}=G\left(x^{+}(u)\right) .
\end{aligned}
$$

where the vector field $G$ does not explicitly depend on $u$ (i.e., $G(x, u)=G(x)$ ).

Remark 6.3. This class of systems has been shown to be equivalent (up to a coordinate change) to the difference map $x_{k+1}=F_{0}\left(x_{k}\right)+B u_{k}$ for a suitable constant matrix $B$. However, it is of interest to exactly specify the proposed control solutions over this class which indeed well approximates larger classes of systems of the form (5).

By construction, the reduced model associated to (13) evolves according to the differential-difference representation

$$
\begin{aligned}
& \eta^{+}=F_{0}(\eta), \quad \eta^{+}:=\eta^{+}(0) \\
& \frac{\partial \eta^{+}(u)}{\partial u}=\bar{G}_{N}\left(\eta^{+}(u)\right)
\end{aligned}
$$

with $\bar{G}_{N}(\eta)=\left[\nabla_{\eta} F_{0}^{-N}(\eta)\right]_{\eta=F_{0}^{N}(\eta)} G\left(F_{0}^{N}(\eta)\right)$. 
Remark 6.4. The reduced dynamics (32) preserves the structure of the original system (31).

The following results specify Theorems 5.1 and 5.2 for this class of systems.

Corollary 6.1. Let (31) verify Assumption 5.1 with quadratic Lyapunov function $V(x)=x^{\top} P x$ and $P>0$; then, the feedback $u_{k}=L_{r}\left(N, \eta_{k}\right)$ solving the D-ILM problem is provided by

$$
L_{r}\left(N, \eta_{k}\right)=\left(\bar{G}_{N}^{\top}\left(\eta_{k}\right) \bar{G}_{N}\left(\eta_{k}\right)\right)^{-1} \bar{G}_{N}^{\top}\left(\eta_{k}\right) G\left(\eta_{k}\right) \gamma\left(\eta_{k}\right) .
$$

Remark 6.5. The prediction-based feedback $u_{k}=F_{0}\left(\gamma\left(\eta_{k}\right)\right)$ does not solve the D-ILM equality.

Corollary 6.2. Let (31) verify Assumption 5.2 with $S(x)=\frac{1}{2} x^{\top} Q x$; then, the reduced model (32) is u-average passive with respect to the output $H(N, \eta)=$ $\bar{G}_{N}^{\top}(\eta) Q$ and the stabilizing passivity-based feedback is provided by

$$
L_{a v}\left(N, \eta_{k}\right)=-\left(I+\frac{1}{2}\left(\bar{G}_{N}^{\top}\left(\eta_{k}\right)\right)^{\top} Q \bar{G}_{N}\left(\eta_{k}\right)\right)^{-1} \bar{G}_{N}^{\top}\left(\eta_{k}\right) Q F_{0}\left(\eta_{k}\right) .
$$

Remark 6.6. The dynamics (24) is of the form (32) so that the coordinate change $z=\operatorname{col}\left(e^{-\frac{1}{2} x_{2}} x_{1}, x_{2}\right)$ transforms the system into the form $z_{k+1}=F_{0}\left(z_{k}\right)+B u_{k}$. Nevertheless, the non quadratic storage function $\tilde{S}(z)=\frac{1}{2}\left(z_{2}^{2}+e^{3 z_{2}} z_{1}^{2}\right)$ prevents from applying Corollary 6.2.

\subsection{Sampled-data systems}

The proposed strategy applies to nonlinear systems issued from sampling whenever the length of the delay is a multiple of the sampling period $\delta$. For this purpose, consider the input-affine system

$$
\dot{x}(t)=f(x(t))+g(x(t)) u(t-\tau)
$$

with $u(t)=u(k \delta)=u_{k}$ for $t \in[k \delta,(k+1) \delta[$ and affected by entire-delay (i.e., $\tau=N \delta$ for some $N \in \mathbb{N}$ ). The sampled-data equivalent-model is provided by

$$
x_{k+1}=F^{\delta}\left(x_{k}, u_{k-N}\right)
$$

with $F^{\delta}\left(x_{k}, u_{k}\right)=\left.e^{\delta\left(\mathrm{L}_{f}+u_{k} \mathrm{~L}_{g}\right)} x\right|_{x_{k}}$ and $F_{0}^{\delta}(x)=e^{\delta \mathrm{L}_{f}} x$. A first analysis and design on this class of time-delay systems was developed in [13] through predictionbased feedback later improved via the notion of Immersion and Invariance. 
Accordingly, an alternative approach to the aforementioned solution is provided by introducing the reduction map (13) in the form

$$
\eta_{k}=\left.e^{\delta\left(\mathrm{L}_{f}+u_{k-N} \mathrm{~L}_{g}\right)} \circ \cdots \circ e^{\delta\left(\mathrm{L}_{f}+u_{k-1} \mathrm{~L}_{g}\right)} \circ e^{-\tau \mathrm{L}_{f}} x\right|_{x_{k}}
$$

through successive application of the Lie exponential operator. One gets that the reduced dynamics (14) is delay free and parametrized by $\delta$ and $\tau=N \delta$; i.e.

$$
F_{r}^{\delta}\left(\tau, \eta_{k}, u_{k}\right)=\left.e^{\tau \mathrm{L}_{f}} \circ e^{\delta\left(\mathrm{L}_{f}+u_{k} \mathrm{~L}_{g}\right)} \circ e^{-\tau \mathrm{L}_{f}} \eta\right|_{\eta_{k}}
$$

or equivalently with $G^{\delta}(\cdot, u)=\int_{0}^{\delta} e^{-s a d_{f+g u}} g \mathrm{~d} s$ and $A d_{F_{0}} I d=e^{-\tau a d_{f}} I d$

$$
\begin{aligned}
& \eta^{+}=F_{0}^{\delta}(\eta), \quad \eta^{+}:=\eta^{+}(0) \\
& \frac{\partial \eta^{+}(u)}{\partial u}=e^{-\tau a d_{f}} G^{\delta}\left(\eta^{+}(u), u\right) .
\end{aligned}
$$

Once the reduction mapping is computed, the control can be designed on the dynamics (37) or equivalently (38) exploiting the exponential form representation which provides a useful way of computing approximate solutions in the form of power expansions in $\delta$ and $\tau$ (see [13] for further details).

\section{Conclusions}

This works extends the notion of reduction to discrete-time and nonlinear dynamics affected by a constant input delay and provides a way of designing the stabilizing feedback based on the properties of the delay-free system associated to the original dynamics. Future works are addressing the extension of this technique to the case of unknown time-delay and larger classes of time-delay systems (e.g., nonlinear systems affected by multi-channel delays and distributed delays).

\section{Acknowledgment}

Mattia Mattioni thanks the Université Franco-Italienne/Università Italo-Francese (UFI/UIF) for supporting his mobility within his PhD program.

\section{References}

[1] O. J. M. Smith, A controller to overcome dead time, Indian Scientist Association in Japan 2 (6) (1959) 28-33. 
[2] Z. Artstein, Linear systems with delayed controls: A reduction, IEEE Transactions on Automatic Control 27 (4) (1982) 869-879.

[3] E. Fridman, Introduction to Time-Delay Systems: Analysis and Control, Systems \& Control: Foundations \& Applications, E. Fridman, 2014.

[4] C. Califano, S. Battilotti, C. H. Moog, On the geometric interpretation of the Polynomial Lie Bracket for nonlinear time-delay systems, in: 2016 IEEE 55th Conference on Decision and Control (CDC), 2016, pp. 555-560.

[5] I. Karafyllis, M. Malisoff, F. Mazenc, P. Pepe (Eds.), Recent Results on Nonlinear Delay Control Systems, Vol. 4 of Advances in Delays and Dynamics, Springer International Publishing, 2016.

[6] M. Krstic, Delay Compensation for Nonlinear, Adaptive, and PDE Systems, Birkhauser, 2009.

[7] R. Sipahi, T. Vyhlídal, S.-I. Niculescu, P. Pepe (Eds.), Time Delay Systems: Methods, Applications and New Trends, Vol. 423 of Lecture Notes in Control and Information Sciences, Springer-Verlag Berlin Heidelberg, 2012.

[8] J. Lei, H. K. Khalil, Feedback linearization for nonlinear systems with timevarying input and output delays by using high-gain predictors, IEEE Transactions on Automatic Control 61 (8) (2016) 2262-2268.

[9] M.-T. Laraba, S. Olaru, S.-I. Niculescu, Analysis of PWA control of discretetime linear dynamics in the presence of variable time-delay, in: Decision and Control (CDC), 2016 IEEE 55th Conference on, IEEE, 2016, pp. 567-572.

[10] I. Karafyllis, M. Krstic, Discrete-time systems, in: Predictor Feedback for Delay Systems: Implementations and Approximations, Springer, 2017, pp. 251-278.

[11] Y. Tian, Y. Cai, Y. Sun, Finite-time stability of discrete-time switched delay systems with nonlinear disturbances, in: 2016 35th Chinese Control Conference (CCC), 2016, pp. 1492-1497.

[12] A. Seuret, F. Gouaisbaut, E. Fridman, Stability of discrete-time systems with time-varying delays via a novel summation inequality, IEEE Transactions on Automatic Control 60 (10) (2015) 2740-2745. 
[13] S. Monaco, D. Normand-Cyrot, M. Mattioni, Sampled-data stabilization of nonlinear dynamics with input delays through Immersion and Invariance, IEEE Transactions on Automatic Control 62 (5) (2016) 2561 - 2567.

[14] I. Karafyllis, M. Krstic, Numerical schemes for nonlinear predictor feedback, Mathematics of Control, Signals, and Systems 26 (2014) 519-546.

[15] S. Monaco, D. Normand-Cyrot, Immersion and Invariance stabilization of nonlinear discrete-time dynamics with delays, in: 2015 54th IEEE Conference on Decision and Control (CDC), 2015, pp. 5049-5054.

[16] S. Monaco, D. Normand-Cyrot, Nonlinear average passivity and stabilizing controllers in discrete-time, Systems \& Control Letters 60 (2011) 431-439.

[17] M. Mattioni, S. Monaco, D. Normand-Cyrot, Further results on sampleddata time-delay systems, in: 20th IFAC World Congress, 2017.

[18] S. Monaco, D. Normand-Cyrot, V. Tanasa, Digital stabilization of input delayed strict feedforward dynamics, in: Proc. 51st IEEE-CDC, Maui, Hawaii, 2012, pp. 7535-7540.

[19] S. Monaco, D. Normand-Cyrot, A unified representation for nonlinear discrete-time and sampled dynamics, Journal of Mathematic, System, Estimation and Control 7 (1995) 50-3.

[20] M. Fliess, D. Normand-Cyrot, A Lie theoretic approach to nonlinear discrete time controllability via ritt's formal differential groups, Systems \& Control Letters 1 (1981) 179-183.

[21] C. Califano, S. Monaco, D. Normand-Cyrot, On the problem of feedback linearization, Systems and Control Letters 36 (1) (1999) 61 - 67.

[22] S. Monaco, D. Normand-Cyrot, M. Mattioni, Stabilization of feedforward discrete-time dynamics through immersion and invariance, in: 2016 American Control Conference (ACC), 2016, pp. 264-269.

[23] F. Mazenc, H. Nijmeijer, Forwarding in discrete-time nonlinear systems, International Journal of Control 71 (5) (1998) 823-835.

[24] M. Mattioni, S. Monaco, D. Normand-Cyrot, Lyapunov stabilization of discrete-time feedforward dynamics, in: 56th IEEE Conference on Decision and Control (CDC), 2017. 